\title{
Correlating Urinary Tract Infection with Patients' Presenting Symptoms and Bacterial Isolation from Urine in Uyo, Nigeria
}

\author{
Onwuezobe Ifeanyi Abraham*, Ubong Etang Ekerenam, Ekuma Agantem Emmanuel \\ Department of Medical Microbiology and Parasitology, Faculty of Clinical Sciences, University of Uyo, Uyo, Nigeria \\ Email address: \\ ifeanyionwuezobe@uniuyo.edu.ng (O. I. Abraham),meetmedicetang@yahoo.com(U. E. Ekerenam), \\ agantemekuma@uniuyo.edu.ng (E. A. Emmanuel) \\ ${ }^{*}$ Corresponding author

\section{To cite this article:} \\ Onwuezobe Ifeanyi Abraham, Ubong Etang Ekerenam, Ekuma Agantem Emmanuel. Correlating Urinary Tract Infection with Patients' \\ Presenting Symptoms and Bacterial Isolation from Urine in Uyo, Nigeria. Science Journal of Clinical Medicine. \\ Vol. 8, No. 3, 2019, pp. 21-27. doi: 10.11648/j.sjcm.20190803.11
}

Received: June 14, 2019; Accepted: July 17, 2019; Published: July 30, 2019

\begin{abstract}
Background: In clinical practice, diagnosis of urinary tract infection (UTI) ideally involves the presence of symptoms and a positive urine culture results. However, due to lack of laboratory facility and where present, delays in getting laboratory result encountered by attending out-patient Physicians, diagnosis are made from presenting symptoms in patients. Empirical treatments are commenced with expectation of positive outcome. Objectives: The aim of this study was to correlate Urinary tract infection with presenting symptoms in patients and laboratory culture results from mid-stream urine samples in Uyo, Nigeria. Materials and Methods: This six months descriptive cross sectional study was carried out in the outpatient Department of the University of Uyo Teaching Hospital in Uyo. Data was obtained through questionnaire administered on 370 consenting outpatients and laboratory culture results from collected midstream urine were analyzed using SPSS software (version 20.0). The predisposing risk factors in these patient were also assessed. The midstream urine samples from the subjects were standardly processed. Cultured urine samples that yielded Gram negative uropathogens were further identified using Microbact 24E (Oxoid, UK), while those that yielded Gram positive uropathogens were identified by the appropriate standard procedures. Results: The presenting symptoms were; discomfort when urinating, urge incontinence (7.6\%), frequent urination $(11.9 \%)$, and cloudy urine with foul smell $(8.1 \%)$, malaise $(23.8 \%)$, fever $(23.5 \%)$, back/flank pain $(3.8 \%)$ and hematuria $(4.6 \%)(\mathrm{P}<0.001)$. The attendant risks factors assessed were age, gender, cleaning method after urination/defecation, contraceptive use, diabetes, past history of UTI and recent catheter use $(\mathrm{P}<0.05)$. Female outpatients had more laboratory confirmed UTI $(25.9 \%)$ than male outpatients (11.5\%). Also revealed is the fact that there is higher prevalence of UTI in female outpatients within the reproductive age group of 28-37 years (43.8\%). Conclusion: In this study, less than $30 \%$ of female patients with presenting symptoms of UTI had positive urine culture test result while only about $12 \%$ of males with these symptoms are culture positive for UTI in Uyo, Akwa Ibom State, Nigeria.
\end{abstract}

Keywords: Urinary Tract Infection, Presenting Symptoms, Culture Test Result, Uyo, Nigeria

\section{Introduction}

Urinary tract infection (UTI) is a common cause of morbidity and mortality affecting all age groups and genders of patients [1]. This infection is caused by uropathogens that invade the urinary tract, the most common being Escherichia coli [2]. Epidemiological studies have shown that about 150 Million people are infected with UTI annually with cost implication of more than 6 Billion US dollars worldwide [3].

The possible causes of UTI are multi-factorial as the infection involves a wide group of clinical syndromes and diseases that vary in epidemiology, aetiology, location and severity of the condition [4]. The infection is exacerbated in people living in developing countries especially those in rural settings due to malnutrition, poor hygiene and low socioeconomic status [5]. 
The Gram-negative aerobic bacilli are the major group of bacterial pathogens that cause UTI with few of Grampositive ones also implicated [6]. However, some viruses, fungi and parasites have also been reported to infect the urinary tract [7]. The bacterial strains, mostly E. coli can cause UTI in both men and women while Staphylococcus saprophyticus have been found to cause UTI mostly in younger women and can also occur secondary to blood-borne infection ${ }^{[2]}$. Others include Klebsiella spp, Pseudomonas aeruginosa, Morganella spp, Enterobacter spp and Proteus mirabilis. Also implicated are Staphylococcus aureus and Enterococcus faecalis [7]. Urinary tract infections in older women and men with indwelling catheter are mostly caused by $P$. aeruginosa and Proteus spp [8]. These bacteria that cause UTI primarily multiply at the opening of the urethra and ascend to the bladder, while some may spread to the kidneys from the blood stream [9].

Urinary tract infection affects females more than males due to factors such as, anatomy of the female urethra, proximity of the genital tract to the urethra, pregnancy, menopause and sexual activity [10]. Other possible risk factors of UTI include diabetes, allergy, obesity, contraceptive use, past history of UTI, enlarged prostate, catheter use and family history [11]. Nevertheless, some patients with urinary tract disorders may present with no symptoms while others may present with general symptoms such as malaise and loss of appetite. But symptoms such as pain in the side (flank), difficulties with urination, painful burning sensation when urinating, the urge to urinate frequently, pain in the flank, discomfort or pressure in the lower abdomen, cloudy urine with foul smell, blood in urine, malaise and fever [12, 13].

However, the assumption that patients presenting with any or all of these symptoms have UTI cannot be ascertained without confirmation with positive laboratory culture result depicted microbiologically by the presence of $10^{5}$ microorganisms per millimeter or more in properly collected midstream samples of urine is confirmatory for UTI $[6,14]$. There is need therefore to locally correlate these presenting symptoms in patients with laboratory culture result for use in clinical diagnosis which will further enhance the commencement of empirical treatment especially in emergency cases while awaiting laboratory result.

\section{Patients and Methods}

This study was carried out over a 6 month period at the University of Uyo Teaching Hospital, a well attended and only tertiary hospital located in the capital city of Akwa Ibom State, Southern Nigeria. Male and female outpatients with provisional diagnosis of UTI from clinical assessment were recruited for the study. A written informed consent was obtained from them prior to their inclusion.

\subsection{Ethical Consideration}

Ethical approval was obtained from the Ethical Review Board of the University of Uyo Teaching Hospital before the study began.

\subsection{Data Collection}

All included participant were interviewed directly with structured questionnaire to collect data about patient's demographics, current health status, clinical history, presenting symptoms of UTI and information related to personal health and hygiene. Some additional information were collected from female outpatients only, such as: sexual activity, pregnancy, use of contraceptive, method of cleaning after urination/defecation, use of hygiene measures from the genital area and menopause. All information from participants was treated with utmost confidentiality.

\subsection{Sample Collection}

Patients were given a sterile universal container and instructed on how to aseptically collect clean catch midstream urine. Female participants were specifically asked to collect midstream urine with the labia held apart, while males were asked not to deep their phallus in the sterile urine container while collecting urine to avoid contamination. The specimens were labelled, transported to the laboratory, and analyzed within 2 hours of collection.

\subsection{Laboratory Analysis of Samples}

\subsubsection{Macroscopic and Microscopic Examination}

The color of all urine samples were first examined macroscopically for presumptive detection of bacteriuria. Microscopic examination of centrifuged urine was done by wet mount preparation. Ten millimeters $(10 \mathrm{mls})$ of midstream urine sample was transferred into a centrifuge tube and centrifuged for 5 minutes at 1,500 rpm. The urine deposits were re-suspended and a drop of a well-mixed sediment was transferred to a glass slide, covered with a cover slip and examined using 10x and 40x objective to quantify the number of white blood cells, red blood cells, epithelial cells, cast and calcium oxalate crystals present for presumptive detection of bacteriuria or pyuria [8].

\subsubsection{Bacterial Culture, Isolation and Colony Counts}

The calibrated loop method was used for culture and isolation of uropathogens. A $0.002 \mathrm{ml}$ (or $1 / 500 \mathrm{ml}$ ) sterile wire loop was used to inoculate urine on standard agar plates and streaked appropriately. The plates were incubated at $37^{\circ} \mathrm{C}$ for 24 hours. After incubation, a count of 25 bacteria colonies (equivalent to $10^{5} \mathrm{cfu} / \mathrm{ml}$ ) or more on the plates was considered positive for UTI (significant bacteriuria), [8, 15].

\subsubsection{Gram Staining}

Lactose fermenting colonies on CLED agar were Gram stained using standard reagents and following standard procedures, to differentiate Gram positive uropathogens from Gram negative ones.

\subsubsection{Biochemical Identification of Bacteria Isolates}

Identification of Gram negative isolates: This was done using Microbact 24E (MB24E) (Oxoid, UK). This is a standardized micro-substrate system in a 96 well microtitre plate consisting of 2 separate substrate strips of 24 tests 
designed for biochemical identification of Enterobacteriaceae and common miscellaneous Gram negative bacilli (MGNB).

Identification of Gram Positive Isolates: Gram positive isolates were subcultured on Blood agar and Bile Esculin Agar. The isolates which included Enterococci and Staphylococci species were first differentiated using catalase test. Coagulase test was also used to differentiate Staphylococcus aureus from Staphylococcus saprophyticus. Growth in Bile Esculin Agar as small dark colonies and in Brain Heart Infusion broth supplemented with $6.5 \% \mathrm{NaCl}$ was confirmatory for Enterococci species. Isolates were quality controlled using $E$. coli (ATCC 29522) strain for Gram-negative organisms and $S$. aureus (ATCC 29523) strain for Gram positive organisms.

\subsubsection{Data Analysis}

All relevant data collected from consenting participants were entered into MS Excel and exported to SPSS software version 20.0 for statistical analysis. The Pearson Chi Square was used for test of association between variables and odd ratio for comparative analysis at $95 \%$ confidence interval. Pvalue $<0.05$ was considered to be statistically significant.

\section{Results}

A total of 370 participants were recruited for this study and $143(38.65 \%)$ of them were female. The ages of participants ranged from 17 to 70 years.

The most common presenting symptoms in outpatients with suspected UTI were malaise $(23.8 \%)$, followed by fever $(23.5 \%)$, frequent urination $(11.9 \%)$ and cloudy urine with foul smell $(8.1 \%)$. There was statistically significant association between presenting symptoms and urine culture test whether positive or negative, $\mathrm{P}<0.001$ [Table 1].
As regards gender, the prevalence of UTI was higher in females $37(25.9 \%)$ than in males $26(11.5 \%)$ [Table 2]. However, in comparing the relationship between gender and age, female within ages 58- 67 years, had highest prevalence of UTI and this age bracket showed statistical significant relationship with UTI. While males within the age group 2837 years had the highest $(33.3 \%)$ UTI prevalence and which also showed statistical significant relationship with the infection [Table 3].

The predisposing factors in the 143 female outpatients recruited, revealed $32(22.4 \%)$ with past history of UTI, $7(4.9 \%)$ were diabetic, $62(43.4 \%)$ were sexually active while $63(44.1 \%)$ were menopausal women. However, only contraceptive use, past history of UTI and cleaning method after urination/ defecation in females, were found to have statistically significant relationship (table 4 ). The predisposing factors in the 227 male outpatients included, past history of UTI 41(18.1\%), 7(3.1\%) were diabetic, and 5(2.2\%) recently had urinary catheterization. These factors were all statistically significant $(\mathrm{P}<0.05)$ for male UTI [Table 5].

The urine culture test results showed, 63(17.0\%) significant growth, $100(27.0 \%)$ insignificant growth while, $207(56.0 \%)$ showed no bacterial growth [Figure 1]. Of the 62 laboratory isolated uropathogens, Gram negative uropathogens had the highest frequency of occurrence 49(79.0\%) with Escherichia coli 19(30.6\%) being the most predominant, followed by Klebsiella pneumoniae $7(11.3 \%)$, Proteus mirabilis 5(8.1\%), Enterobacter agglomerans complex 5(8.1\%) and Pseudomonas aeruginosa 3(4.8\%). Gram positive uropathogens accounted for only $13(21.0 \%)$ of the isolates and the most predominant was Staphylococcus aureus 6(9.7\%) [Figure 2].

Table 1. Clinical Characteristics of outpatients with UTI.

\begin{tabular}{|c|c|c|c|c|c|c|}
\hline Clinical Symptoms & Urine culture test & No $(\%)$ & Yes $(\%)$ & $\mathbf{X}^{2}$ & P-value & OR $(95 \% \mathrm{CI})$ \\
\hline \multirow[t]{3}{*}{ Discomfort when urinating } & Negative & $301(98.0)$ & $6(2.0)$ & \multirow[t]{3}{*}{81.21} & \multirow[t]{3}{*}{$<0.001^{*}$} & 1 \\
\hline & Positive & $41(65.1)$ & $22(34.9)$ & & & $26.19(10.30-70.29)$ \\
\hline & Total & $342(92.4)$ & $28(7.6)$ & & & \\
\hline \multirow{2}{*}{ Urge Incontinence } & Positive & $34(54.0)$ & $29(46.0)$ & \multirow{2}{*}{105.37} & \multirow{2}{*}{$<0.001^{*}$} & $28.24(12.34-64.62)$ \\
\hline & Total & $342(92.4)$ & $28(7.6)$ & & & \\
\hline \multirow[t]{2}{*}{ Back/Flank Pain } & Negative & $305(99.3)$ & $2(0.7)$ & \multirow[t]{2}{*}{48.59} & \multirow[t]{2}{*}{$<0.001^{*}$} & 1 \\
\hline & Total & $356(96.2)$ & $14(3.8)$ & & & \\
\hline \multirow[t]{3}{*}{ Frequent Urination } & Negative & $298(97.1)$ & $9(2.9)$ & \multirow[t]{3}{*}{138.15} & \multirow[t]{3}{*}{$<0.001^{*}$} & 1 \\
\hline & Positive & $28(44.4)$ & $35(55.6)$ & & & $41.39(18.07-94.79)$ \\
\hline & Total & $326(88.1)$ & $44(11.9)$ & & & \\
\hline \multirow[t]{3}{*}{ Cloudy Urine with foul smell } & Negative & $304(99.0)$ & $3(1.0)$ & \multirow[t]{3}{*}{123.05} & \multirow[t]{3}{*}{$<0.001^{*}$} & 1 \\
\hline & Positive & $36(57.1)$ & $27(42.9)$ & & & $76.00(21.95-263.10)$ \\
\hline & Total & $340(91.9)$ & $30(8.1)$ & & & \\
\hline \multirow[t]{2}{*}{ Hematuria } & Negative & $304(99.0)$ & $3(1.0)$ & \multirow[t]{2}{*}{53.82} & \multirow[t]{2}{*}{$<0.001^{*}$} & 1 \\
\hline & Total & $353(95.4)$ & $17(4.6)$ & & & \\
\hline \multirow[t]{3}{*}{ Fever } & Negative & $273(88.9)$ & $34(11.1)$ & \multirow[t]{3}{*}{155.11} & \multirow[t]{3}{*}{$<0.001^{*}$} & 1 \\
\hline & Positive & $10(15.9)$ & $53(84.1)$ & & & $42.56(19.82-91.36)$ \\
\hline & Total & $283(76.5)$ & $87(23.5)$ & & & \\
\hline \multirow[t]{3}{*}{ Malaise } & Negative & $271(88.3)$ & $16(11.7)$ & \multirow[t]{3}{*}{144.6} & \multirow[t]{3}{*}{$<0.001$} & 1 \\
\hline & Positive & $11(17.5)$ & $52(82.5)$ & & & $35.59(17.02-74.40)$ \\
\hline & Total & $282(76.2)$ & $88(23.8)$ & & & \\
\hline
\end{tabular}

* Statistically significant at $\mathrm{P}<0.001, \mathrm{X}^{2}=$ Chi-square, $\mathrm{OR}=$ Odd Ratio at $95 \%$ confidence interval (CI). 
Table 2. Prevalence of Urinary Tract Infection in relation to gender.

\begin{tabular}{|c|c|c|c|c|c|}
\hline Gender & No. of samples Examined & No. of positive Samples (\%) & $x^{2}$ & P-value & Odd Ratio \\
\hline Male & 227 & $26(11.5)$ & & & 1 \\
\hline Female & 143 & $37(25.9)$ & 12.91 & $0.0001^{*}$ & 2.7 \\
\hline Total & 370 & $63(17.0)$ & & & \\
\hline
\end{tabular}

*Statistically significant, $\left(x^{2}(1)=12.91 ; \mathrm{P}=0.0001 ; \mathrm{OR}=2.7\right)$

Table 3. Prevalence of UTI in Outpatients in relation to age and gender.

\begin{tabular}{|c|c|c|c|c|c|c|}
\hline \multirow{2}{*}{$\begin{array}{l}\text { Age group } \\
\text { (years) }\end{array}$} & \multicolumn{3}{|l|}{ Male } & \multicolumn{3}{|l|}{ Female } \\
\hline & No. Examined & UTI positive '十ve' cases (\%) & P-value & No. Examined & UTI positive '+ve' cases (\%) & P-value \\
\hline $16-27$ & 72 & $5(6.9)$ & & 14 & $2(14.3)$ & \\
\hline $28-37$ & 24 & $8(33.3)$ & & 32 & $14(43.8)$ & \\
\hline $38-47$ & 30 & $3(10.0)$ & م 000 * & 7 & $2(28.6)$ & * \\
\hline $48-57$ & 60 & $3(5.0)$ & $0.0005^{\circ}$ & 68 & $10(14.7)$ & $0.01^{*}$ \\
\hline $58-67$ & 31 & $5(16.1)$ & & 17 & $8(47.1)$ & \\
\hline$\geq 68$ & 10 & $2(20.0)$ & & 5 & $1(20.0)$ & \\
\hline
\end{tabular}

*Statistically significant

Table 4. Risks Factors Predisposing Female Outpatients to UTI ( $n=143)$.

\begin{tabular}{|c|c|c|c|c|c|c|c|}
\hline \multirow{2}{*}{ Parameters } & \multirow{2}{*}{ Response } & \multicolumn{2}{|c|}{ Urine Culture (Females) } & \multirow[b]{2}{*}{ Total (\%) } & \multirow[b]{2}{*}{ P-value } & \multirow[b]{2}{*}{ OR } & \multirow[b]{2}{*}{$95 \%$ CI } \\
\hline & & Negative (\%) & Positive (\%) & & & & \\
\hline \multirow{2}{*}{ Past history of UTI } & No & $94(84.7)$ & $17(15.3)$ & 111(77.6) & \multirow{2}{*}{$>0.001^{*}$} & 1 & \multirow{2}{*}{$(4.09-24.7)$} \\
\hline & Yes & $12(37.5)$ & $20(62.5)$ & $32(22.4)$ & & 10.05 & \\
\hline \multirow{2}{*}{ Diabetes } & No & $103(75.7)$ & $33(24.3)$ & $136(95.1)$ & \multirow{2}{*}{0.06} & 1 & \multirow{2}{*}{ (1.09-35.64 } \\
\hline & Yes & $3(42.9)$ & $4(57.1)$ & $7(4.9)$ & & 6.2 & \\
\hline \multirow{2}{*}{ Allergy } & No & $87(77.0)$ & $26(23.0)$ & $113(79.0)$ & \multirow[b]{2}{*}{0.91} & 1 & \multirow{2}{*}{$(0.32-3.68)$} \\
\hline & Yes & $19(63.3)$ & $11(36.7)$ & $30(21.0)$ & & 1.07 & \\
\hline \multirow{2}{*}{ menopause } & No & $62(77.5)$ & $18(22.5)$ & $80(55.9)$ & \multirow{3}{*}{0.27} & 1 & \multirow{3}{*}{$(0.72-3.23)$} \\
\hline & Yes & $43(68.3)$ & $20(31.7)$ & $63(44.1)$ & & 1.52 & \\
\hline \multicolumn{6}{|l|}{ Contraceptive use: } & & \\
\hline \multirow{2}{*}{ Diaphragm } & No & $99(77.3)$ & $29(22.7)$ & $128(89.5)$ & \multirow{2}{*}{$<0.001^{*}$} & 1 & \\
\hline & Yes & $0(0.0)$ & $15(100.0)$ & $15(10.5)$ & & & \multirow{3}{*}{$(1.68-8.99)$} \\
\hline \multirow{2}{*}{ SSC } & No & $74(80.4)$ & $18(19.6)$ & $92(64.3)$ & \multirow{2}{*}{$0.01^{*}$} & 1 & \\
\hline & Yes & $34(66.7)$ & $17(33.3)$ & $51(35.7)$ & & 3.88 & \\
\hline \multicolumn{8}{|l|}{ Sexual Activity: } \\
\hline \multirow{2}{*}{ Active } & No & $63(77.8)$ & $18(22.2)$ & $81(56.6)$ & \multirow{2}{*}{0.23} & 1 & \multirow{2}{*}{$(0.75-3.36)$} \\
\hline & Yes & $43(69.4)$ & 19(30.6) & $62(43.4)$ & & 1.58 & \\
\hline \multirow{2}{*}{ Not active } & No & $43(69.4)$ & $19(30.6)$ & $62(43.4)$ & 023 & 1 & $\left(\begin{array}{llll}0 & 30-1 & 34\end{array}\right)$ \\
\hline & Yes & $63(77.8)$ & $18(22.2)$ & $81(56.6)$ & 0.25 & 0.63 & $(0.50-1.04)$ \\
\hline Hygiene measures in genital & No & $69(77.5)$ & $20(22.5)$ & $89(62.2)$ & 021 & 1 & $(076-349)$ \\
\hline area: FS & Yes & $37(68.5)$ & $17(31.5)$ & $54(37.8)$ & 0.21 & 1.63 & $(0.76-3.49)$ \\
\hline Powder & No & $100(73.5)$ & $36(26.5)$ & $136(95.1)$ & 0.95 & 1 & \\
\hline & Yes & $6(85.7)$ & $1(14.3)$ & $7(4.9)$ & 0.95 & 0.56 & $(0.06-4.92)$ \\
\hline Douching & No & $43(70.5)$ & $18(29.5)$ & $61(42.7)$ & 0.54 & 1 & $(0.34-155)$ \\
\hline & Yes & $63(76.8)$ & $19(23.2)$ & $83(57.3)$ & 0.54 & 0.73 & $(0.34-1.55)$ \\
\hline Cleaning method: & & & & & & & \\
\hline Front to back & No & $4(12.1)$ & 29(87.9) & $33(23.1)$ & $<0001^{*}$ & 1 & 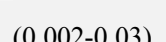 \\
\hline Front to Dack & Yes & 102(92.7) & $8(7.3)$ & $110(76.9)$ & $<0.001$ & 0.01 & $(0.002-0.03)$ \\
\hline Back to front & No & $102(92.7)$ & $8(7.3)$ & $110(76.9)$ & $<000 *$ & 1 & $(30714046)$ \\
\hline Back to front & Yes & $4(12.1)$ & $29(87.9)$ & $33(23.1)$ & $<0.001$ & 123.3 & $(30 . / 1-494.6)$ \\
\hline
\end{tabular}

*Statistically significant, $\mathrm{OR}=\mathrm{odd}$ ratio, $\mathrm{SSC}=$ spermicidal-coated condom, $\mathrm{FS}=$ feminine spray

Table 5. Risks Factors Predisposing Male Outpatients to UTI ( $n=227)$.

\begin{tabular}{llllllll}
\hline Urine Culture (Males) & & & & & & \\
\hline Parameters & Response & Negative (\%) & Positive (\%) & Total (\%) & P-value & OR & 95\% CI \\
\hline Past history of UTI & No & $175(94.1)$ & $11(5.9)$ & $186(81.9)$ & $<0.001^{*}$ & 1 & \\
& Yes & $26(63.4)$ & $15(36.6)$ & $41(18.1)$ & & 8.84 & $(3.67-21.25)$ \\
Diabetes & No & $201(91.4)$ & $19(8.6)$ & $220(96.9)$ & $<0.001^{*}$ & & \\
& Yes & $0(0.0)$ & $7(100.0)$ & $7(3.1)$ & & & \\
Recent catheter removal & No & $201(90.5)$ & $21(9.5)$ & $222(97.8)$ & $<0.001^{*}$ & \\
& Yes & $0(0.0)$ & $5(100.0)$ & $5(2.2)$ & & \\
\hline
\end{tabular}

*Statistically significant, $\mathrm{OR}=$ odd ratio, $\mathrm{CI}=$ confidence interval 


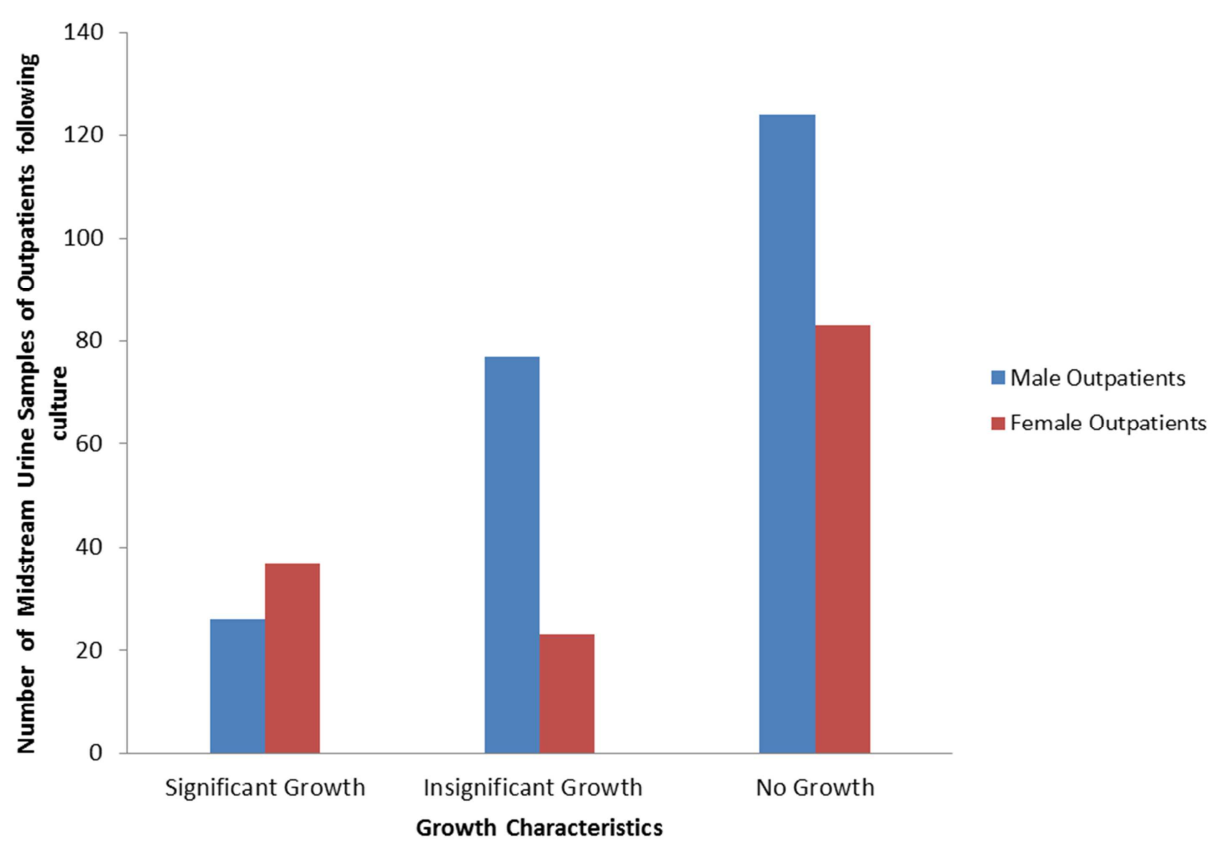

Figure 1. Results of Culture of Midstream Urine Samples of Outpatients $(n=370)$.

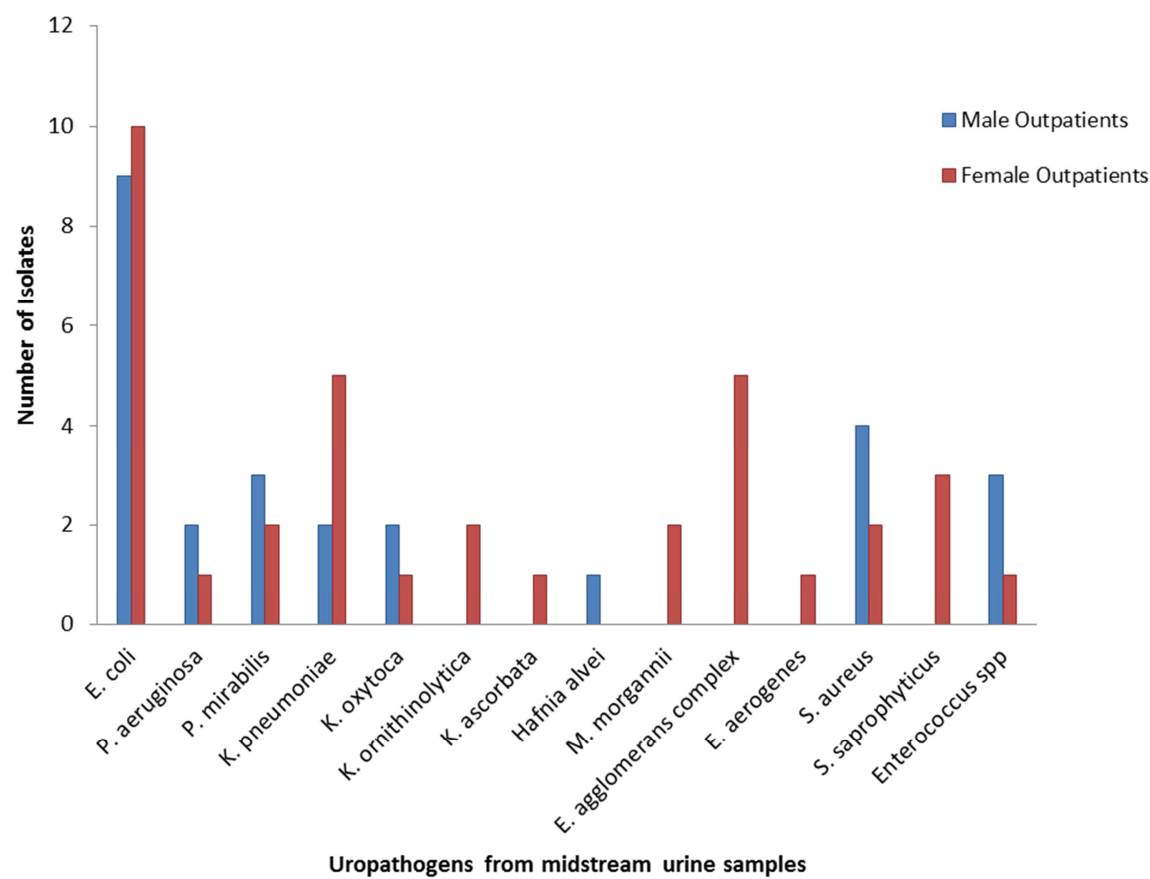

Figure 2. Distribution of uropathogens isolated from midstream urine samples of outpatients in relation to gender ( $n=62$ ).

\section{Discussion}

Urinary tract infection is one of the most common infections that occur in both the community and hospital settings due to the invasion of the urinary tract by microbial pathogens. Acute uncomplicated urinary tract infection is known to be characterized by a combination of symptoms such as dysuria, urinary frequency, urge incontinence, gross hematuria, fever, flank pain and discomfort with pyuria [7]. In this study, the prevalence of the presenting symptoms was higher in laboratory culture positive proven UTI than culture negative UTI. There was indeed a statistical significant association between UTI and presenting clinical symptoms $(p<0.001)$, an indication that these symptoms are reflective of the infection.

The presenting symptoms in Patients that were most associated with bacteria positive urine culture result was fever $(84.1 \%)$ followed by malaise $(82.5 \%)$ and frequent urination $(55.6 \%)$. These were consistent with some study reports locally and internationally $[16,17,18]$. Those with presenting symptoms of; flank pains $(81 \%)$, discomfort while urinating $(65.1 \%)$ and urge incontinence $(54 \%)$ respectively, have less bacteria positive urine culture result when 
compared to those without these symptoms. These presenting symptoms were found also to have significant association with UTI prevalence. This shows that the majority of patients suffering from UTI will not present with these symptoms.

Pyuria which presents in patients as cloudy or turbid urine, but microscopically as the presence of $\geq 10$ white blood cells (WBCs) per high power field [8], was observed in 80 urine samples, out of which 63(78.8\%) yielded significant bacterial growth at a prevalence rate of $17.0 \%$. This is a lower rate when compared to studies from Lagos and Nassarawa States in Nigeria, which recorded $35.5 \%$ and $60 \%$ prevalence rates respectively $[19,20]$. This possibly may be due to inhibition of growth by antibiotic [21], taken by patients before presentation which is a common practice in our environment.

The prevalence of laboratory confirmed UTI in this study was higher in females than in males. This has generally been known to be so due to the peculiarities of the shortness of the female urethra. However, as regards the prevalent predisposing risk factors in female outpatients from this study, past history of UTI, contraceptive use and anal cleaning methods after defecation were found to be statistically significant. Of special note is the deviation from general held belief that that female anal cleaning method of front to back after defeacation helps in reducing UTI when compared to cleaning from back to front. This study revealed equal risks for both methods. These practices may have constituted part of personnel hygiene reported in other studies. However isolating these practices have become necessary in this study as patients and health personnel alike have associated the occurrence of UTI in females with especially the cleaning method of back to front. Active sexual activity is not a statistically significant risk factor for UTI in females in this study. This probably explains the statistically significant and higher prevalence of laboratory confirmed UTI among the older female age group of $58-67$ years, rather than the younger more sexually active age groups [22].

The prevalent risk factors which were statistically significant for UTI in males were past history of UTI, diabetes mellitus and recent catheter removal. The male age group $28-37$ years was found to be statistically significant age for UTI. This is the sexually active age group and with the other aforementioned risk factors, is found to be in agreement with study by Foxman [23, 24].

Among the male patients, predisposing risk factors such as past history of UTI (18.1\%) diabetes $(3.1 \%)$, and recently removed urinary catheter $(2.2 \%)$ were found to have statistical significantly association with UTI causation in them $(\mathrm{P}<0.05)$ [25].

The Gram-negative bacilli $49(79.0 \%)$ class of bacteria were highly prevalent when compared to Gram-positive bacteria especially cocci $13(21.0 \%)$. Among the Gram negative bacterial isolates, were Escherichia coli (30.6\%) which was the most prevalent uropathogen followed by Klebsiella pneumoniae (11.3\%). Staphylococcus aureus (9.7\%), was the most prevalent Gram positive cocci. These results were consistent with the reports of another author [17] which revealed $85.0 \%$ of Gram-negative bacilli and $15.0 \%$
Gram-positive cocci of which E. coli and Klebsiella $s p$ were the most prevalent bacterial agents respectively. The result also agrees with similar work done by some authors $[20,26$, 27], but differs from reports of others [28, 29] which recorded Pseudomonas aeruginosa and Klebsiella sp; and Alcaligens sp and Klebsiella aerogenes respectively as the most prevalent uropathogens.

More Staphylococcus aureus 4(6.5\%) were isolated from male outpatients with fever than in female outpatients 2(3.2\%). Staphylococcus aureus has been implicated as the cause of descending UTI especially in patients with associated fever [7]. However, this finding is less than what was reported from a similar study [29] which reported higher $S$. aureus in both male $(19.1 \%)$ and female outpatients $(6.7 \%)$. The reason for this disparity may not be unconnected with antibiotic use before presentation to the hospital.

Of interest, is the isolation of Hafnia alvei and Klebsiella ornithinolytica in this study which has not been so as confirmed by the records of the study center. Hafnia alvei is a known opportunistic pathogen and has been reported to be found in urine cultures of patients with underlying predisposing immune suppressing illness [30]. This may be so as some of the patients had diseases such as diabetes with the probable attendant immune depression in these patients. Also, the isolation of these $K$. Ornithinolytica formerly known as ornithine-positive $K$. Oxytoca and $H$. alvei were made possible due to the use of a more sensitive biochemical identification system, Microbact 24E (Oxoid, UK) which is not routinely used in most hospital laboratories.

\section{Conclusion}

The majority of outpatients presenting with symptoms of urinary tract infection in Uyo do not have Microbiological confirmed UTI as only about $30 \%$ of female patients with presenting symptoms of UTI had positive urine culture test result while only about $12 \%$ of males with these symptoms are culture positive for UTI in Uyo, Akwa Ibom State, Nigeria.

Furthermore, patients with suspected UTI presents with various symptoms among which are fever, malaise, discomfort while urinating, urge incontinence, flank pain, frequent urination, cloudy urine with foul smell and haematuria. These symptoms were found to have statistical significant association with culture proven UTI. However, more than $80 \%$ of the patients that complained of fever and malaise had culture proven UTI, and as such, making these symptoms not only the much sought clinical symptoms in malaria infection but also of UTI in a resource poor environment like Nigeria.

\section{References}

[1] P. Suwangool (2012). Current Management of Urinary Tract Infections. Bangkok Medical Journal, 3, 101-106.

[2] P. Anuja and M. D. Shah (2015). Overview of Urinary Tract Symptoms. Merck Manual: Kenilworth, USA. 
[3] C. M. Gonzalez and A. J. Schaeffer (1999). Treatment of Urinary Tract Infection: what's old, what's new and what works. World Journal of Urology, 17 (6):372-382.

[4] M. J. Lucas and F. G. Cunningham (1993). Urinary Tract Infections in Pregnancy. Clinical Obstetrics and Gynaecology, 36 (4): $855-868$.

[5] S. M. Ahmed and A. K. Avazara (2008). Urinary Tract Infections (UTI) Among Adolescent Girls in Karimnagar District. Indian Journal of Medicine, 39, 12-15.

[6] L. B. Travis And B. H. Bruhard (1991). Infections of the Urinary Tract. In: Rudolph's Paediatrics, $19^{\text {th }}$ edn. Stamford, Appleton and Lange, p. 237.

[7] R. H. Rubin, T. R. Jr. Beam and W. E. Stamm (1992). An Approach to Evaluating Antibacterial Agents in the Treatment of Urinary Tract Infection. Clinical Infectious Diseases Journal; 14 (supplement 2): S253-254.

[8] M. Cheesbrough (2010). District Laboratory Practice in Tropical Countries, part 2, New York, USA: Cambridge University Press, pp. 62-118.

[9] K. Gupta, T. M. Hooton, K. G. Naber, B. Wullt, R. Colgan and L. G. Miller (2011). International Clinical Practice Guidelines for the Treatment of Acute Uncomplicated Cystitis and Pyelonephritis in Women: A 2010 update by the Infectious Diseases Society of America and the European Society for Microbiology and Infectious Diseases. Clinical Infectious Diseases, 52 (5): 103-120.

[10] A. J. Schaeffer, N. Rajan and Q. Cao (2001). Host Pathogenesis in Urinary Tract Infections. International Journal of Antimicrobial Agents, 17 (4):245-251.

[11] A. L. Flores-Mireles, J. N. Walker, M. Caparon and S. J. Hultgren (2015). Urinary Tract Infections: Epidemiology, Mechanisms of Infection and Treatment Options. Nature Reviews Microbiology, 13, 269-284.

[12] K. Gupta and B. Trautner (2012). Urinary Tract Infections in the Clinic. Annal of International Medicine, 156 (5): 1-15.

[13] J. D. Sobel and D. Kaye (2010). Urinary Tract Infections. In: Mandell, Douglas \& Bennett's Principles of Infectious Diseases, G. L. Mandell, J. E. Bennett \& R. Dolin, Eds., Churchill Livingstone, Philadelphia, PA: USA, pp. 957-985.

[14] N. E. Tolkoff and R. H. Rubin (1986). Urinary Tract Infection. Significance and Management. Bulletin of the New York Academy of Medicine, 62 (2): 131-148.

[15] H. D. Isenberg (2012). Urine Culture. In: Clinical Microbiology Handbook. Pakistan Antimicrobial Resistance Network, pp. 1-31.

[16] D. Clayson, D. Wild, H. Doll, K. Keating and K. Gondek (2005). Validation of a Patient-administered Questionnaire to Measure the Severity and Bothersomness of Lower Urinary Tract Symptoms in Uncomplicated Urinary Tract Infection (UTI): The UTI Symptom Assessment Questionnaire. British Journal of Urology International; 96, 350-359.

[17] B. B. Oluremi, A. O. Idowu and J. F. Olaniyi (2011). Antibiotic Susceptibility of Common Bacterial Pathogens in Urinary Tract Infections in a Teaching Hospital in South Western Nigeria. African Journal of Microbiology Research, 5 (22): 3658-3663.
[18] P. K. C. Arul, K. G. Dileesh and M. Vijayan (2012). A Cross Sectional Study on Distribution of UTI and their Antibiotic Utilisation Pattern in Kerala. International Journal of Research in Pharmaceutical and Biomedical Sciences, 3 (3): 1125-1130.

[19] K. O. Akinyemi, S. A. Alabi, M. A. Taiwo and E. A. Omonigbehin (1997). Antimicrobial Sussceptibility Pattern and Plasmid Profiles of Pathogenic Bacteria Isolated from Subjects with Urinary Tract Infections in Lagos, Nigeria. Niger Quarterly Journal of Hospital Medicine, 1, 7-11.

[20] A. S. Kolawole, O. M. Kolawole, Y. T. Kandaki-Olukemi, S. K. Babatunde and K. A. Durowade (2009). Prevalence of Urinary Tract Infections (UTI) among Patients Attending Dalhatu Araf Specialist Hospital, Lafia, Nasarawa State, Nigeria. International Journal of Medicine and Medical Sciences, 1 (5): 163-167.

[21] E. E. Okonfua and B. N. Okonofua (1989). Incidence and Pattern of Asymptomatic Bacteriuria of Pregnancy in Nigeria Women. Nigerian Medical Practitioner, 17, 354-358.

[22] R. Raz, Y. Gennesin, J. Wasser, Z. Stoler, S. Rosenfeld, E. Rottensterich and W. E. Stamm (2000). Recurrent urinary tract infections in postmenopausal women. Clin Infect Dis. 30 (1):152-6.

[23] Foxman, B. (2002). Epidemiology of UTIs: Incidence, Morbidity and Economic Cost. American Journal of Medicine, 113 Supplement 1A, 5S-13S.

[24] B. Foxman, P. Somsel, P. Tallman, B. Gillespie, R. Raz, and R. Colodner et al. (2001) Urinary tract infection among women aged 40 to 65: behavioral and sexual risk factors. J Clin Epidemiol. 54 (7):710-8.

[25] Z. Huang, H. Xiao, H. Li, W. Yan and Z. Ji (2017). Analysis of the incidence and risk factors of male urinary tract infection following urodynamic study. Eur J Clin Microbiol Infect Dis. 36 (10):1873-1878.

[26] H. I. Inabo and H. B. T. Obanibi (2006). Antimicrobial Susceptibility of some Urinary Clinical Isolates to commonly used Antibiotics. African Journal of Biotechnology, 5 (5): 487-489.

[27] A. O. Kehinde, K. S. Adedapo, C. O. Aimaikhu, A. A. Odukogbe, O. Olayemi and B. Salako (2011). Symptomatic Bacteriuria among Asymptomatic Antenatal Clinic Attendees in Ibadan, Nigeria. Tropical Medicine and Health, 39 (3): 7376.

[28] O. A. Aboderin, A. Abdu, B. W. Odetoyinbo and A. Lamikanra (2009). Antimicrobial Resistance in Escherichia coli Strains from Urinary Tract Infections. National Medical Association, $101,1268-1273$.

[29] F. D. Otajevwo (2012). Urinary Tract Infection among Symptomatic Outpatients Visiting a Tertiary Hospital Based in Mid-Western Nigeria. Global Journal of Health Sciences, 5 (2): 187-199.

[30] P. R. Murray, F. C. Tenovar, A. P. Michael, E. I. Baron, M. A. Pfaller and R. H. Yolken (1995). Manual of Clinical Microbiology, $6^{\text {th }}$ ed. American Society for Microbiology, Washington, DC, pp. 231-236. 